BÚSQUEDA - Julio / Diciembre de 2015 - No. 15 (30 - 37)

\title{
Estrategias de prevención y tratamiento de la fascitis plantar en adultos
}

\author{
Elena Conde Pascual ${ }^{1}$, Michael Peralta Bustamante ${ }^{2}$, Cesar Guerra Monterroza ${ }^{1}$, Deimer David Sierra \\ Martínez ${ }^{1}$
}

Recibido: 16-07-2015 - Aceptado: 14-09-2015

\begin{abstract}
Resumen
La fascitis plantar se define como una patología dolorosa del retropié localizada en la parte inferomedial del talón y suele ser la causa más frecuente de dolor en esta región en la población adulta. Es habitual que se presente debido una vida sedentaria y en personas con sobrepeso. El aumento de la edad es otro factor de riesgo añadido. Se produce una pérdida de elasticidad, disminución de la fuerza en los músculos intrínsecos, trastornos biomecánicos en el pie y/o una mayor incapacidad de regenerar los tejidos tisulares. Si se produce después de los 40 años, la causa más probable puede ser la disminución de la almohadilla de grasa en el talón inferior, que provoca en consecuencia, la reducción de la absorción de los impactos y de la protección del calcáneo. Los tratamientos que se pueden aplicar en este tipo de lesión son los que incluyen crioterapia, vendaje funcional, ortesis plantares, férulas nocturnas, calzado adecuado, el tratamiento físico y manual los cuales son, estiramientos, ejercicios de fortalecimiento, infiltraciones con corticoides y la acupuntura; también es importante el tratamiento con ultrasonidos, ondas de choque extracorpóreas, los impulsos eléctricos de baja frecuencia y por último se puede aplicar la cirugía para lo que será la recuperación total de esta lesión. El objetivo de este artículo es conocer los antecedentes relacionados con la fascitis plantar en adultos, el cual está centrado en un estudio de revisión bibliográfica, ya que se buscaron referencias relacionadas con el tema de interés. Se concluyó que la fascitis plantar es una lesión que afecta a gran porcentaje de personas, en especial a los adultos a partir de los 40 años de edad, aunque también esta puede presentarse en la población en general y puede ser tratada con base en métodos que sirven para aliviarla o mejorarla total o parcialmente.
\end{abstract}

Palabras clave: Lesiones, pie, tratamiento, fascitis plantar, adultos.

\footnotetext{
${ }^{1}$ Doctora en Ciencias de la Actividad Física y del Deporte. Corporación Universitaria del Caribe (CECAR).

${ }^{2}$ Licenciados en Ciencias del Deporte y la Actividad Física. Corporación Universitaria del Caribe (CECAR).
} 
Elena Conde Pascual, et al., - Estrategias de prevención y tratamiento de la fascitis plantar en adultos

\title{
Strategies of prevention and treatment in adults plantar fasciitis
}

\begin{abstract}
Plantar fasciitis is defined as a painful hindfoot pathology located in the inferomedial of the heel and usually the most common cause of pain in this region in the adult population. It is usual to occur in people who are sedentary or overweight individuals. Increasing age is another risk factor added. A loss of elasticity, decreased strength in the intrinsic muscles, biomechanical foot disorders and / or increased tissue inability to regenerate tissues occurs. If it occurs after age 40 , the most likely cause is the decrease in fat pad in the lower bead, causing a consequent reduction of shock absorption and protection of the calcaneus. Treatments that can be applied in this type of injury, are those that include cryotherapy, taping, plantar orthotics, night splints, proper footwear, physical therapy and manual which are stretching, strengthening exercises, and corticosteroid injections Acupuncture is also important sonication, extracorporeal shock wave, the low frequency electrical impulses and finally surgery can apply for what will be a full recovery from this injury. The aim of this paper was to determine the background associated with plantar fasciitis in adults, which is centered on a study of literature review as references related to the topic of interest were sought. It was concluded that the plantar fasciitis is an injury that affects a large percentage of people, especially adults from 40 years of age, although this may occur in the general population and can be treated based on methods they serve to alleviate or improve totally or partially.
\end{abstract}

Keywords: Injuries; foot; treatment; adults.

\section{Introducción}

Los estudios relacionados con la fascitis plantar (FP) tuvieron su origen en 1812 por Wood, quien la atribuyó a una patología relacionada con la tuberculosis. Cuando se desacreditaron las teorías infecciosas, en 1957 se pensó que el origen de esta patología se debía al atrapamiento de la fascia debido a la presencia de un espolón calcáneo. Posteriormente se pensó que la aparición de este se debía a la tracción continuada de la fascia, con lo que se descartó que la causa fuera el propio espolón (Martínez, 2013; Neufeld, 2008). Maio (1993) afirma que la talalgia plantar o fascitis plantar consiste en una degeneración de las fibras de colágeno de la aponeurosis fibrosa que proporciona soporte a la bóveda plantar y amortigua las fuerzas que se ejercen sobre ella.

La fascitis plantar es la afección más frecuente de dolor no traumático en el tobillo-pie. Se presenta más en mujeres entre los 40 y 70 años con comienzo progresivo y difuso en planta del pie y tobillo que poco a poco se agudiza impidiendo la marcha (Díaz López y Guzmán Carrasco, 2014). La "fascitis plantar" o también llamada "talalgia plantar" se define como una patología dolorosa del retropié localizada en la parte inferomedial del talón y suele ser la causa más frecuente de dolor en esta región en la población adulta. Es posible definirla como "una inflamación del tejido conectivo grueso que se encuentra en la planta del pie y que se fija en el talón" (Alcántara, 2006).

Se trata de una lesión multifactorial secundaria a diversos factores de riesgo biomecánicos, medioambientales y anatómicos. Se caracteriza por una sobrecarga mecánica de la fascia plantar que produce un dolor localizado en la zona antero-interna del talón (Chana, 2013; Torrijos, 2009; Dyck, 2004; Puttaswamaiah, 2007; Buchbinder, 2004; Riddle, 2003). Aunque a veces es frecuente oír el término "espolón calcáneo" para referirse a esta patología, actualmente lo más aceptado es "fascitis plantar" que alude a una fase inflamatoria aguda o "fasciosis plantar" que es propiamente una degeneración crónica.

La fascitis plantar es muy común observarla en atletas y corredores de todos los niveles afecta, aproximadamente, al $10 \%$ de ellos 
BÚSQUEDA - Julio / Diciembre de 2015 - No. 15 (30 - 37)

durante su carrera deportiva. En la población general, se produce también en un porcentaje similar, sobre todo en personas en las que su profesión les requiera estar largos periodos de tiempo en bipedestación y/o con carga de peso. En la mayoría de los casos, la fascitis plantar, es un proceso auto limitado y la resolución de los síntomas se produce entre los 10 y 12 meses de media (Torrijos, 2009; Dyck, 2004; Buchbindert; 2004, Kibler, 1991). También es habitual que se presente en personas que llevan una vida sedentaria o sufran sobrepeso, ya que la obesidad, aparte de aumentar el nivel de discapacidad del paciente, incrementa el riesgo de padecer fascitis plantar (Puttaswamaiah, 2007; Riddle, 2003). Así mismo, personas que trabajen con grandes cargas o personas que presenten anomalías biomecánicas en el pie tendrán más posibilidades de padecerlo (Puttaswamaiah, 2007; Riddle, 2003).

Así pues, la FP suele afectar, en especial, a los corredores, pero puede impedirse siguiendo consejos de prevención y evitando las posibles causas que pueden producirla. En los casos ya instaurados, los deportistas deberán modificar su actividad deportiva, buscando un plan de entrenamiento alternativo, ya que aunque sea necesario un reposo relativo, deberán mantener la forma física y el rendimiento aeróbico (Juliano, 2004; Kibler, 1991).

Según los distintos estudios la fascitis plantar puede presentarse por distintas causas en ser; idiopática, mecánica, anatómica, biomecánica, tisular y extrínseca. La idiopática puede ser posiblemente multifactorial; la mecánica por permanecer periodos largos de tiempo en bipedestación o soportando grandes cargas de peso; la anatómica consiste en problemas de pie varo, valgo, equino, cavo y obesidad, la biomecánica consiste en trastornos biomecánicos del pie disminución de la dorsiflexión del tobillo y exceso de pronación mantenida, la tisular; consiste en cambios degenerativos del tejido adiposo en el talón, disminución progresiva de colágeno, agua y elasticidad de la aponeurosis plantar y por último, la extrínseca; debido superficies duras o calzados inadecuados
(Rosenbaum, 2014; Chana, 2013; Martínez, 2013; Torrijos, 2009; Dyck, 2004; Riddle, 2003).

Con la edad se produce una pérdida de elasticidad en la aponeurosis plantar, disminución de la fuerza en los músculos intrínsecos, trastornos biomecánicos en el pie y/o una mayor incapacidad de regenerar los tejidos tisulares. Si se produce después de los 40 años, la causa más probable sea la disminución de la almohadilla de grasa en el talón inferior, junto con la pérdida de agua, colágeno y tejido elástico, que provocan como consecuencia la reducción de la absorción de los impactos y de la protección del calcáneo (Rosenbaum, 2014; Ayub, 2005; Dyck, 2004). Otras de las causas que pueden producir FP son la disminución de dorsiflexión del tobillo y el exceso de pronación mantenida del pie, pues esto aumenta el estrés del tejido fascial. Los factores que pueden provocar esto son el calzado inadecuado, una intensidad mayor en las actividades de la vida diaria o las alteraciones anatómicas del pie, como el pie varo, valgo, equino, plano y cavo (Rosenbaum, 2014; Neufeld, 2008; Ayub, 2005). A la hora de realizar el abordaje terapéutico, es importante que la educación forme parte de ello. El buen conocimiento y compresión por parte del paciente permitirá escoger la mejor opción de tratamiento, ya que existe una gran variedad de opciones de tratamiento, algunas de ellas sin clara evidencia científica (Martínez, 2013; Buchbinder, 2004).

Existen múltiples tratamientos que pueden ser aplicados en este tipo de lesión, como lo son los conservadores que incluyen crioterapia, vendaje funcional, ortesis plantares, férulas nocturnas, calzado adecuado, el tratamiento físico y manual como lo son, estiramientos, ejercicios de fortalecimiento, infiltraciones con corticoides y la acupuntura; también es importante el tratamiento de electro terapia que lleva consigo los ultrasonidos, ondas de choque extracorpóreas, electrolisis percutánea intratisular, y los impulsos eléctricos de baja frecuencia y por último; se puede aplicar la cirugía y los factores de crecimiento plaquetario para lo que será la recuperación total de esta lesión. (Martínez, 2013; 
Buchbinder, 2004). El tratamiento de la fascitis plantar en los últimos 15 años ha tenido una importante evolución; los distintos estudios dan a conocer algunos de los tratamientos más eficaces que se utilizaron o se están utilizando a la hora de tratar dicha patología. Según autores como Crosby (2001) y Núñez (2000), se debe utilizar un calzado adecuado; blando y con un ligero tacón sin que sea excesivo y su suela debe ser gruesa para que absorba los impactos. En este sentido, otros estudios proponen el tratamiento a través de infiltraciones con corticoides, con el que se reduce el dolor, pero solo a corto plazo, ya que si no se corrige la disfunción biomecánica que lo provoca, el problema no se solucionará (Tallia, 2003).

A través de los años han ido surgiendo nuevos métodos, al observarse debilidad en la musculatura intrínseca de los flexores de los dedos y de la musculatura extensora (Wearing, 2004). Cuando existen estas debilidades se emplean los ejercicios de fortalecimientos concéntricos para la mejora de la resistencia y fuerza de los músculos (Rosenbaum, 2014; Torrijos, 2009; Alshami, 2008; Dyck, 2004; Bolgla, 2004; Kibler, 1991). Otro tipo de tratamiento utilizado es el de las férulas nocturnas que se usan frecuentemente como tratamiento de la FP, ya que estas mantienen el tobillo en posición neutra durante la noche lo que impide la retracción de la fascia, sin embargo las personas que las usan abandonan e interrumpen el tratamiento, según afirma el estudio (Martin, 2005).

Coughlin (2007) y Núñez (2000) dan a conocer en su escrito que la cirugía sería la última solución a la cual se debería recurrir, dado el caso que los tratamientos anteriores no hayan sido eficaces. La técnica consiste en una liberación completa de la fascia plantar, extirpando las zonas degeneradas y eliminando el espolón calcáneo si se encuentra presente. Posteriormente se realizará una inmovilización postoperatoria con yeso durante un mínimo de 3 semanas y después se implantará una plantilla de soporte (Coughlin, 2007; Núñez, 2000).

En el año 2009 se da a conocer el tratamiento con ultrasonidos donde se interviene este tipo de lesión, este procedimiento se aplicará a las 48 horas de la lesión, en la fase post aguda. Los ultrasonidos disminuirán el dolor y el espasmo muscular. Además, aumentará el riego sanguíneo y el metabolismo (Torrijos, 2009; Kamel, 2000). Hacia el año 2010 los estudios afirman que la acupuntura, como tratamiento de la fascitis plantar, puede modificar la sintomatología dolorosa, mejorando las funciones en otras estructuras corporales (Hernández, 2010). Haciendo referencia a otro tipo de tratamientos, con el uso de las ondas de choque extracorpóreas se afirma que se obtiene una mejoría significativa después de tres meses de seguimiento, siempre que se combinen con plantillas semirrígidas individualizadas. Por sí solas, las ondas de choque extracorpóreas no experimentan una mejoría significativa, además su efecto secundario más frecuente es el dolor (Martínez, 2013; Thomas, 2010). Por otra parte, los estiramientos se muestran como una terapia más para la mejora de la problemática planteada, y tienen como objetivo principal mejorar la tensión tisular mediante un estiramiento controlado e indoloro de la fascia por parte del paciente. Los estiramientos se realizarán de forma lenta y no supondrán una gran dificultad para que el paciente pueda realizarlos. Se emplean para incrementar la extensibilidad de los tejidos blandos a través de efectos mecánicos (Martínez, 2013; Torrijos, 2009; DiGiovanni, 2003).

Según estudios realizados, el uso de ortesis plantares individualizadas semirrígidas haresultado ser más eficaz que el uso de plantillas blandas individualizadas o blandas prefabricadas para disminuir el dolor (Martínez, 2013; Walther, 2013). En este mismo año se empleó otro tratamiento con impulsos eléctricos de baja frecuencia el cual consiste en unos impulsos voltaicos controlados por software (PBK-2C). Sin embargo, entre los 3 y 6 meses de seguimiento no existe diferencia significativa con respecto al dolor, movilidad dorsiflexora del tobillo y grosor de la fascia plantar entre los grupos experimentales (Chana, 2013).

Cabe resaltar la crioterapia como un tratamiento reciente que consiste en el uso del frío, el cual se emplea en una fase aguda del 
BÚSQUEDA - Julio / Diciembre de 2015 - No. 15 (30 - 37)

dolor, como cualquier otra patología inflamatoria. Se pueden usar bolsas de hielo-gel o cualquier bolsa congelada de una forma más casera. Actualmente existen aparatos especiales para focalizar la crioterapia. El frío disminuirá el dolor, la inflamación y el hematoma. Además será el tratamiento más eficaz después de correr o al acabar el día (Rosenbaum, 2014; Torrijos, 2009; Neufeld, 2008).

En este trabajo se analizan las características propias de la fascitis plantar, así como también las causas, pautas de prevención y su tratamiento con diversas técnicas expresadas por los autores consultados durante la realización de este trabajo, los distintos métodos que se utilizaron o se están utilizando a la hora de intervenir para tratar esta patología como tal. El objetivo del presente artículo es conocer los estudios realizados sobre la problemática de la lesión, la evolución de las técnicas y tratamiento empleados para la mejora de la misma. Este estudio tiene como utilidad recopilar información relevante de cara a poder conocer de manera más amplia la problemática estudiada.

\section{Metodología}

Se trata de una revisión bibliográfica que analiza y discute resultados y hallazgos de otros estudios acerca de la fascitis plantar. Se realizó una búsqueda minuciosa y muy puntual utilizando palabras claves como lo fueron fascitis, fascia plantar, fascitis plantar, talalgia.

Se estudiaron 40 artículos y se tomaron los más relacionados al tema aquí tratado, los cuales sirvieron para realizar el análisis pertinente de dicha lesión.

Para la localización de los antecedentes o documentos bibliográficos se utilizaron varias fuentes documentales como Scopus, ScienceDirect, ProQuest.

También se realizó una búsqueda en internet en el buscador "Google académico" con los mismos términos.

\section{Discusión}

Maio (1993) afirma que la talalgia plantar o fascitis plantar consiste en una degeneración de las fibras de colágeno de la aponeurosis fibrosa que proporciona soporte a la bóveda plantar y amortigua las fuerzas que se ejercen sobre ella. Unos años más tarde Kwong (1998) definió la fascitis plantar como un síndrome resultante de traumatismos repetitivos en la cara plantar de la fascia en su origen.

Alcántara (2006) define la fascitis plantar como una patología dolorosa del retropié localizada en la parte inferomedial del talón y suele ser la causa más frecuente de dolor en esta parte del pie en la población adulta. Estudios posteriores afirman que la fascitis plantar es la causa más común de dolor en el talón, y consiste en la inflamación de la fascia plantar, a nivel de la tuberosidad del calcáneo. Produce un dolor intenso localizado y que puede irradiarse a toda la planta del pie (Gómez, 2012).

Otros estudios destacan que se trata de una lesión multifactorial secundaria a diversos factores de riesgo biomecánicos, medioambientales y anatómicos. Se caracteriza por una sobrecarga mecánica de la fascia plantar que produce un dolor localizado en la zona antero-interna del talón (Chana, 2013; Torrijos, 2009; Dyck, 2004; Puttaswamaiah, 2007; Buchbinder, 2004; Riddle, 2003). Una investigación publicada en Medlineplus en el año (2012), sostiene que la fascitis plantar se observa tanto en hombres como en mujeres; sin embargo, casi siempre afecta a los hombres activos entre los 40 y 70 años y es una de las dolencias ortopédicas más comunes relacionadas con el pie. Mientras que para Díaz y Guzmán (2014) se presenta más en mujeres entre los 40 y 70 años con comienzo progresivo y difuso en planta del pie y tobillo, que poco a poco se agudiza, impidiendo la marcha.

Diferentes autores (Torrijos, 2009; Dyck, 2004; Buchbindert; 2004, Kibler, 1991) afirman que la fascitis plantar es muy común observarla en atletas y corredores de todos los niveles $y$, afecta, aproximadamente, al 10\% de ellos 
Elena Conde Pascual, et al., - Estrategias de prevención y tratamiento de la fascitis plantar en adultos

durante su carrera deportiva. En la población general, se produce también en un porcentaje similar, sobre todo en personas en las que su profesión les requiera estar largos periodos de tiempo en bipedestación y/o con carga de peso. En la mayoría de los casos, la fascitis plantar, es un proceso auto limitado y la resolución de los síntomas se produce entre los 10 y 12 meses. En el estudio realizado por García (2012) acerca de lesiones de las partes blandas del pie, se menciona que la fascitis plantar suele presentarse en pacientes obesos de 40 a 60 años que pasan largo tiempo de pie o caminan en superficies duras. En otro estudio realizado por el facultativo especialista en rehabilitación del hospital universitario fundación Alcorcón de Madrid, García (2007) junto con sus colaboradores establecen que la fascitis plantar puede aparecer en atletas, profesionales o no, en personal militar y en la población general sedentaria.

Con respecto a la eficacia de los estiramientos en la recuperación de la fascitis plantar existen tres estudios que orientan sobre cuáles son los estiramientos más efectivos en esta patología. DiGiovanni (2003 y 2007) comparó la eficacia de estiramientos de gemelo y sóleo frente a estiramientos de la fascia plantar y encontró que estos obtenían mejores resultados, aunque ambos grupos conseguían reducir el dolor y mejorar la autonomía de los pacientes. Por otro lado, Radford (2007) estudió la eficacia a corto plazo de los estiramientos del tríceps sural (14 días después de la aplicación) y no encontró una mejora en los pacientes.

Hay diversos trabajos que han estudiado la respuesta de la fascitis plantar a diferentes tratamientos. Los tratamientos conservadores para la fascitis plantar tienen éxito en el 80-90\% de los pacientes (Wolgin, 1991). Según Tatli y Kapasi (2009) cuando el tratamiento conservador no tiene éxito la inyección con esteroides es el tratamiento más recomendable. Por otra parte Bordelo (1993) encontró que el 95\% de los pacientes tratados en forma conservadora responden al tratamiento dentro de 6 a 10 meses.

\section{Conclusiones}

Se puede concluir que la fascitis plantar afecta un número elevado de personas adultas, sobre todo, a partir de los 40 años de edad. Para recuperarse de esta lesión, pueden aplicarse diferentes métodos que sirven para aliviarla o mejorarla total o parcialmente. Para el tratamiento de la fascitis plantar se han descrito muchas intervenciones con una gran variedad de técnicas fisioterápicas, siendo más frecuente la combinación de más de una de estas técnicas. Su efectividad ha resultado demostrada en la mayoría de los casos. La velocidad de recuperación de la fascitis plantar se cree que aumenta con el inicio del tratamiento en las primeras 6 semanas de aparición de los síntomas, sin embargo, esto aún no ha podido ser demostrado. Debido a que la combinación de varias técnicas puede ser más efectiva, no se recomienda la aplicación de un solo tratamiento para la recuperación, sino que es necesario elegir correctamente el tratamiento que más se ajuste a la etiología de la fascitis del paciente.

Los tratamientos más recientes y con mayor auge son las técnicas de la electrólisis percutánea intratisular o EPI, esta técnica consiste en la aplicación de una corriente galvánica mediante una aguja de acupuntura directamente en el lugar de la lesión. El objetivo es desnaturalizar y eliminar el tejido degenerado, para así favorecer la regeneración de los tejidos blandos y tendones de forma natural. Para ello se utiliza una maquinaria específica que es la encargada de generar corriente galvánica de baja intensidad, y que tiene un aplicador con el que se coloca la aguja de acupuntura estéril. Otra técnica que se utiliza en la actualidad es la del ultrasonido que consiste en eliminar el tejido enfermo y dejar espacios en el que pueda crecer un nuevo tejido, utilizando para ello imágenes de ultrasonidos; tras dos semanas de tratamiento se puede observar una mejoría en los síntomas de dicha lesión. Todos estos tratamiento actuales apuntan a la más pronta recuperación de la persona para volver a su vida cotidiana lo antes posibles. 
BÚSQUEDA - Julio / Diciembre de 2015 - No. 15 (30 - 37)

\section{Referencias bibliográficas}

Alcántara, S., Ortega, E., García, F., Sánchez, I., Ferrero, A., y Aguilar, J. J. (2006). En: dolor de tobillo y pie. Manual serme de rehabilitación y medicina física. Madrid: Ed Médica Panamericana, 445-51.

Alshami AM, Souvlis T, Coppieters MW. (2008). A review of plantar heel pain of neural origin: differential diagnosis and management. Man Ther 13,103-111.

Ayub A, Yale SH, Bibbo C. (2005). Common foot disorders. Clin Med Res 3, 116-119.

Bolgla LA, Malone TR. (2004). Plantar fasciitis and the windlass mechanism: a biomechanical link to clinical practice. J Athl Training 39, $77-82$

Buchbinder R. (2004). Clinical Practice, Plantar Fasciitis. N Engl J Med 350, 21592166.

Chana P. (2013). Eficacia de los impulsos eléctricos de bajo voltaje en la fasciosis plantar (tesis doctoral). Ed. UCM, Madrid.

Coughlin MJ, Mann RA, Saltzman CL. (2007). Surgery of the foot and ankle. Ed. Mosby Elsevier, Philadelphia, PA, pp. 689-705.

Crosby W, Humble RN. (2001). Rehabilitation of plantar fasciitis. Clin Pod Med Surg 18, 225-231.

DiGiovanni BF, Nawoczenski DA, Lintal ME, Moore EA, Murray JC, Wilding GE, Baumhauer JF. (2003). Tissue-specific plantar fasciastretching exercise enhances outcomes in patients with chronic heel pain. A prospective, randomized study. J Bone Joint Surg Am 85, 1270-1277.

Dyck DD Jr, Boyajian-O'Neill LA. (2004). Plantar fasciitis. Clin J Sport Med 14, 305.

García Estrada, E. M., Álvarez Cambras, R., Rodríguez Vázquez, M. I., Valdés Díaz, A., y González Fundora, N. (2005). Fascitis plantar tratada con ondas de choque extracorpóreas. Revista cubana de ortopedia y traumatología, 19(1), 0-0.

García-Santos J. (2003). Infiltraciones locales en Atención Primaria (III). Miembro inferior. SEMERGEN 29, 17-27.

Hernández AP, Campos DM, Pescador Y. (2010). Intervencion fisioterapeutica a traves de la acupuntura, para el manejo del dolor en fascitis plantar. Ed. UMB, Colombia, pp. 31-38.

Juliano PJ, Harris TG. (2004). Plantar fasciitis, entrapment neuropathies and tarsal tunnel syndrome: current up to date treatment. Curr Opin Orthop 15, 49-54.

Kamel M, Kotob H. (2000). High frequency ultrasonographic findings in plantar fasciitis and assessment of local steroid injection. $J$ Rheumatol 27, 2139-2141.

Kibler W, Goldberg C, Chandler T. (1991). Functional biomechanical deficits in running athletes with plantar fasciitis. Am J Sports Med 19, 66-71.

Martin RL, Irrgang JJ, Burdett RG, Conti SF, Van Swearingen JM. (2005). Evidence of validity for the Foot and Ankle Ability Measure (FAAM). Foot Ankle Int 26, 968-983.

Martínez JA. (2013). Ortesis plantares rígidas conformadas y ondas de choque extracorpóreas en el tratamiento de la fascitis plantar (tesis doctoral). Ed. UM, Murcia.

Núñez M, Llanos LF. (2000). Biomecánica, medicina y cirugía del pie. Ed: Masson; Barcelona.

Puttaswamaiah R, Chandran P. (2007). Degenerative plantar fasciitis: a review of current concepts. The Foot 17, 3-9.

Riddle D, Pulisic M, Pidcoe P, Johnson RE. (2003). Risk Factors for plantar fascitis: a matched case-controlled study. J Bone Joint Surg Am 85-A, 872-877. 
Rosenbaum AJ, DiPreta JA, Misener D. (2014). Plantar heel pain. Med Clin North Am. 98, 339-352.

Sánchez JM. (2010). Fascitis plantar: tratamiento regenerativo mediante electrólisis percutánea intratisular $(\mathrm{EPI} \cap)$. Podología Clínica. 2, 22-29.

Tallia AF, Cardone DA. (2003). Diagnostic and therapeutic injection of the ankle and foot. Am Fam Phys 68, 1356-1362.

Thomas JL, Christensen JC, Kravitz SR, Mendicino RW, Schuberth JM, Vanore JV, Weil LS Sr, Zlotoff HJ, Bouché R, Baker J. (2010). American College of Foot and Ankle Surgeons Heel Pain Committee. The diagnosis and treatment of heel pain: a clinical practice guideline-revision 2010. J Foot Ankle Surg 49, 1- S19.
Torrijos A, Abián-Vicén J, Abián $P$, Abián M. (2009). Plantar fasciitis treatment. J Sport Health Res 1,123-131.

Walther M, Kratschmer B, Verschl J, Volkering C, Altenberger S, Kriegelstein S, Hilgers $M$. (2013). Effect of different orthotic concepts as first line treatment of plantar fasciitis. Foot Ankle Surg 19,103-107.

Wearing SC, Smeathers JE, Yates B, Sullivan PM, Urry SR, Philip D. (2004). Saggital movement of the medial longitudinal arch is unchanged in plantar fasciitis. Med Sci Sports Exerc 36, 1761-1767. 